This case was six months and a half advanced. The child was living, and died in 28 hours.

We have means now within our power, with which in former years we were unacquainted,-the ergot for instance; but perhaps there may have been unfortunate results from incautious use of it; and very satisfactory effects in many cases may, I think, be obtained more safely from opium. Mrs. O., in December last, is an example.

June 25, $185 \mathrm{I}$.

\section{A CASE OF}

NON-UNION OF FRACTURE OF BOTH BONES OF THE LEG, OF EIGHTEEN WEEKS' STANDING, SUCCESSFULLY TREATED BY MERCURY.

By R. TIIURSfield, Esq., M.R.C.S., Broseley. SENIOR-BURGEON TO IRONBRIDGE DISPEXSARY.

IN the month of August, 1847, I was asked to see an old patient of mine, aged 42 , who had twice suffered severely from acute rheumatism. I learnt from her that about eighteen weeks before, she had, on returning from an evening visit to her sister, slipped in crossing a rough road, and fractured both bones of the leg just above the ankle. She was not far from her sister's house, and a surgeon was soon in attendance. Her leg was placed on a pillow and the usual means employed; but in about six weeks after the accident it was ascertained that no bony union had taken place. Upon further advice starch bandages were applied; but no benefit resulted. On examining the leg I found it greatly swollen, shorter by an inch at least than the other, with a good deal of deformity, greatly increased by any attempt at standing upon it, and the least motion at the point of fracture. She had greatly increased in bulk during the confinement she had undergone, but considered herself in good health.

I was a good deal puzzled what plan to pursue with the best chance of successfully relieving her, for I had never had to treat such a case before, and the uncertain results in the experience of others were anything but encouraging. In thinking over the case I remembered that the late Mr. Tyrrell had said mercury in his hands had proved at least as efficacious as any other plan he had tried; and I made up my mind to use it, because the peculiarly irritable state of the patient put sawing off the ends of the bone, seton, or any other surgical means quite out of the question, and most others seemed to have been fairly tried. I therefore procured two very strong gutta percha splints, which reached from rather above the knee to the bottom of the foot, and having, on the 18th, soaked them in boiling water, I made a complete cast of the leg by passing a tight roller over them and allowing them to cool. Having brought the ends of the bones as nearly into apposition as I could and covered the parts with mercurial ointment, \&c., after the plan recommended by Mr. John Scott, I applied the splint, and bound all together with a long elastic bandage. I confined her to bed, and tied the leg on a pillow, giving her five grains of blue pill night and morning. No perceptible effect from the mercury took place until she had taken sixteen pills, when suddenly violent ptyalism set in, which lasted a fortnight. On the 7 th of October the bandages, \&c., were removed, as they had become loose from the reduced size of the leg, and a great improvement was found to have taken place The leg was longer, the deformity less, and the motion at the fracture scarcely perceptible. Greatly encouraged by such a state of things, I renewed the applications exactly as at first, and allowed her to use a sofa during the day. In about three weeks I again tightened the roller, and in another month removed everything from the leg, and found the fracture perfectly united and firm. For a month after this time she had the leg well rubbed night and morning with camphorated oil, to get rid of the remaining œdema, wearing a roller and one splint during the day, to protect the leg if she should chance to slip in walking. In six months she could walk well two or three miles, and is now as active as most women of her age, the broken leg being as useful as the other.

\section{CASES IN PRIVATE PRACTICE.}

\section{By JAMES KITSELL, Esq., Droitwich.}

Read at the Nineteenth Anniversary Meeting, held at Brighton,August, 1851.

Case I.-Case of Retention of Urine, with False Passage, and Paralysis of Bladder.

Is March, 1850, I was requested to attend J. P., a farmer of independent means, who had laboured under retention of urine at intervals for nearly a week. On my arrival, the medical man in attendance, " although not qualified," had made numerous attempts, unsuccessfully, to introduce the catheter; a medical gentleman from Birmingham having also visited the case twice, and succeeded in introducing the catheter, though not without great loss of blood, owing in some measure to the unskilful use of the instrument, before his arrival, and the formation of a false passage, which, considering the age of the patient, $(69$,$) who was of impaired$ constitution, rendered the case very serious. On my arrival I learnt from the patient that he had long been subject to dribbling of urine, frequent inc'ination to void it, and other symptoms indicative, at lis period of life, of prostatic enlargement, of which I satisfied myself on examination per rectum. On introducing the catheter it readily entered the false passages, and no little manœuvring was required to conduct it into the bladder. The instrument being of No. 5 size, passed with difficulty through the constricted prostatic portion if the "rethra, when about two pints of urinn were 
drawn off, the size of the catheter being gradually increased until No. 12 could be passed with little difficulty. Owing to the over-distended state of the bladder at the onset of the case, when more than three pints of urine were removed, this viscus became so paralyzed, that nearly seven weeks elapsed before it evinced any symptoms of resuming its tone, during which time the patient felt not the slightest inclination to void his urine, although a pint or more would occasionally be collected, owing to the patient's residence being a distance of nearly five miles, and my having to visit him twice daily. The treatment consisted in the internal and external use of strychnia, iodide of potassium, with Plummer's pill and conium at bed-time, and keeping the catheter occasionally in the bladder through the night.

Suffice it to state that the patient recovered, and voided his urine better than he had done for some years, until he experienced a slight return about fifteen months after, owing, as I suspect, to some excitement of the genital organs, from causes which I need not here attempt to explain, which was easily relieved by timely aid.

I have merely reported this case to show how much relief can be afforded in a case which appeared for some time nearly hopeless, since there was great reason to fear that the tone of the bladder should be irrecoverably lost.

\section{Case II.-Extensive Fracture of the Ribs, with Emphysema.}

A porter, employed at the Droitwich station of the railway, was proceeding to fasten the connecting chain of a luggage train, which contained thirty-six tons behind and sixteen in front of him, when, on the train being suddenly moved, in his endeavour to escape, ha became pinned between the beam ends, (if I may so term them,) there being no spring buffers as in passenger trains. Being frightfully crushed, he was brought to Droitwich, (a distance of a mile and a half,) in the omnibus, in a state of collapse, which lasted for some time, and from which it was not expected that he would rally; however, under the cautious use of diffusible stimuli, he slowly rallied, a quantity of blood and frothy saliva issuing from his mouth. As reaction proceeded, acute inflammation of the lung succeeded, the 2nd, 3rd, 4th, 5th, and 6th ribs on the left side being sadly fractured in their sternal and dorsal aspects. As the power of respiration increased, a tumour, larger than a distended sheep's bladder, occupied the seat of injury over the lung, the laceration in which must have been considerable, since this tumour appeared to become directly inflated at each respiration, alternately rising and falling with remarkable impulse, until more than one-half the body was inflated; several punctures having been made into the over-distended cellular membrane, and a bandage applied as soon as practicable, with copious depletion, the patient being of stout robust constitution, considerable difficulty was experienced with the rising end of the 2nd rib, which from its position could not be readily compressed with a bandage, to obviate which difficulty I employed a spring truss, on Salmon and Ody's principle, which, passing over the shoulder, the requisite compression was effected by the anterior pad, and the fractured ends thus kept in apposition. Considerable quantities of pus streaked with blood were expectorated from this extensive wound in the lung, but fortunately the patient recovered, and at the end of six weeks resumed his employment, which he has continued for very many months, apparently as well as before the accident. The crepitus during the act of respiration was audible at a considerable distance from the patient.

\section{Case III.-Instantaneous Relief in a Case of Croup from Fright.}

M. D., aged 9 years, was suddenly attacked with symptoms of acute croup, the peculiar cough, difficult respiration, with the head thrown back, and threatening symptoms of suffocation being apparent, it was deemed advisable to abstract blood as usual, when the child's great objection to the application of leeches threw her into a regular paroxysm of rage and fright, which, producing a regular and copious perspiration, all the symptoms immediately vanished, no return being experienced. An equal objection or dread of the lancet was evinced on an attempt being made to open the jugular vein. This case merely shows what good can be effected through the medium of the skin under peculiar circumstances; the shock given to the system, in addition to the copious perspiration, must have had its share in reducing the vascular and nervous influence, and thus removing a complaint which, under ordinary treatment, might have proved dangerous or fatal.

\section{Case IV.-Diabetes in an Infant.}

It will appear strange that after the case of hydrocephalus in one of my own children another still more peculiar and interesting-nay unparalleled, should occur in my own domestic circle, viz., diabetes; the subject being a male child now nearly eight months old, who, although born plump and apparently healthy, began to manifest, "a few days after birth," symptoms of diabetes, the quantity of urine voided being enormous, and truly characterized as diabetes mellitus, the smell and colour of honey being so strong as first of all to attract the attention of the mother and nurse, who, without any idea of the complaint, observed to me that the napkins smelt fragrant like honey, which remark, in fact, led me to the case, the nature of which was now (about a fortnight after birth) too evident to be mistaken; the usual concomitant symptoms being strongly developed, viz.,-a hot dry skin, voracious appetite, constipation of the bowels, extreme emaciaciation; in fact, the little patient may be termed a living skeleton. It is really wonderful that he should have survived so long in this state, having been almost in statu quo for nearly six months; the cranial bones being nearly as moveable as when first born. Although troubied with cough, apparently the result of repeated catarrhal attacks, there is no decided pulmonic disease, 
as he cries remarkably strong, nor is there any hectic tendency apparent in the countenance by night or day, the catarrhal attacks being induced, most probably, by the change of temperature to which the body is exposed, consequent on the frequent change of napkins by night and day, (which average about one per hour,) completely soaked. It is remarkable that not the slightest acidity has been traceable in this case, as connected with the stomach.

The food consists partly of the breast-milk and partly of panada, or bread boiled in water to the consistence of thick custard, sucked from the glass feedingbottle with India-rubber teat, which food the child unfortunately refuses unless sweetened. As regards the treatment in so young a subject, great disadvantage prevails, inasmuch as anything in the form of medicine is speedily rejected, and occasionally the food, apparently from over-distension, the result of that insatiable desire on the part of the stomach to become over-loaded; and when not satisfied in this way his cries are distressing. Milk food, beef-tea, and such like, are also rejected, no attempt having at present been made to convey it by means of enemata. The preparations of iron, the Ferri Sesquicarb, and Tinct. Ferri Sesquichloridi, could only be administered at intervals, when diarrhœa occasionally, although rarely, occurred, which medicines in cases of a cachectic tendency, and with an impoverished state of the blood, deprived of its carbonate of iron, I have found from experience most beneficial; an occasional dose of Hydrarg. cum Cretâ, or calomel, being sometimes required, owing to the deficiency of bile in the alvine excretions; opiates being sometimes needed to procure rest. Dover's powders, with diaphoretic doses of Antim. Tartarizatum, appeared most suitable; but the stupefaction consequent on any medicine of this kind, in careful doses, soon prohibited their administration. Cod-liver oil was tried, with a view to its nutrient property, but was soon rejected. As this disease has been considered by authors to depend on a shattered state of the constitution, and peculiar to spirit drinkers, or to an excess of vegetable diet, and in the decline of life, this case becomes at once instructive and interesting, inasmuch as no such cause could have influenced the subject of this report. In conclusion, I may observe that the urine, although not diminished in quantity, has lost, in a great measure, its melliferous property, being now of a more cadaverous or putrescent smell, a similar odour being observable in the alvine excretions. I have not tested the urine with reference to any albuminous property which it may possess, nor have I yet tried the phosphoric acid.

\section{Remarks on the Present Poor-Law.}

Mr. Kitsell then called the attention of the meeting to the present inadequate remuneration of the Poor-Law medical staff, who are still not half paid in their annual salary, besides having much to contend with in reference to any claim they may have for extras, which are but partially provided for in the $P \ldots \ldots$ antinne. for instance, in the scale of allowances for fractures, dislocations, and midwifery, no allowance is made by the guardians for fractures of the clavicle, jaw, ossa nasi, \&c. The guardians will not allow for fractures of the clavicle, although strictly a portion of the upper extremity, because the term arm is used in the printed form of allowances. And in fractured ribs, even with emphysema, the fee has been refused, becalise they are not specified. And in cases of bad wounds, scalds, burns, \&c., which must of necessity accasionally fall into the hands of the union surgeon, no provision is made. In cases of midwifery, when the maximum fee of $£ 2$ is demanded for the operation of turning in placenta, arm, or other preternatural presentations, as also in forceps and other instrumental cases, the medical officer has, (after inquiries made by the relieving officer of the nurse and patient, anything but pleasant or delicate,) to explain the particulars of his operation and proceeding to the Board, who ultimately decide (for want of knowing better) that such a case is not entitled to the maximum fee, and reduce it to $10 \mathrm{~s}$., the medical officer being lucky if he gets occasionally $£ 1$ for an operation which rescues his patient from the jaws of death, independently of riding some forty or fifty miles in after-attendance on some of those cases. No allowance at all being made in cases of abortion or premature labour, unless the pregnancy be sufficiently advanced to admit of the child's being born alive. Surely there needs reform in this as in other departments of Poor-Law medical remuneration. In cases of abortion much more trouble, anxiety, and attendance are required than in ordinary cases at the full period, especially when these cases happen in the country. It is much to be regretted that members of an honourable and enlightened profession should be subject to the caprice and decision of Boards of Guardians, who are composed of good, bad, and indifferent, quite incompent to judge of medical matters, or the remuneration connected therewith.

\section{Result of the Case of Hydrocephalus.}

Mr. Kitsell also stated that his child, on whom he operated for hydrocephalus, in April, 1849, reported at the Seventeenth Anniversary Meeting, (Provincial Journal, August 8th, 1846, page 431) continues to enjoy uninterrupted good health.

\section{DR. THOMSON'S INVALID LIFTER.}

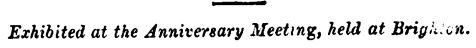

THIs apparatus, of which the following wood-cut gives an excellent representation, has been constructed for the purpose of raising and removing from bed, and into an adjoining room if required, heavy and unwieldy patients, reduced to a state of helplessness by disease or 